\title{
3-D Respiratory Motion Compensation during EP Procedures by Image-Based 3-D Lasso Catheter Model Generation and Tracking
}

\author{
Alexander Brost ${ }^{1}$, Rui Liao ${ }^{2}$, Joachim Hornegger ${ }^{1}$, and Norbert Strobel $^{3}$ \\ 1 Chair of Pattern Recognition, Department of Computer Science, \\ Friedrich-Alexander-University Erlangen-Nuremberg, Erlangen, Germany \\ Alexander.Brost@informatik. uni-erlangen.de \\ 2 Siemens Corporate Research, Imaging and Visualization, Princeton, NJ, USA \\ 3 Siemens AG, Healthcare Sector, Forchheim, Germany
}

\begin{abstract}
Radio-frequency catheter ablation of the pulmonary veins attached to the left atrium is usually carried out under fluoroscopy guidance. Two-dimensional X-ray navigation may involve overlay images derived from a static pre-operative 3 -D volumetric data set to add anatomical details. However, respiratory motion may impair the utility of static overlay images for catheter navigation. We developed a system for image-based 3-D motion estimation and compensation as a solution to this problem for which no previous solution is yet known. It is based on 3 -D catheter tracking involving 2-D/3-D registration. A biplane X-ray $\mathrm{C}$-arm system is used to image a special circumferential (lasso) catheter from two directions. In the first step of the method, a 3-D model of the device is reconstructed. 3-D respiratory motion at the site of ablation is then estimated by tracking the reconstructed model in 3-D from biplane fluoroscopy. In our experiments, the circumferential catheter was tracked in 231 biplane fluoro frames (462 monoplane fluoro frames) with an average $2-\mathrm{D}$ tracking error of $1.0 \mathrm{~mm} \pm 0.5 \mathrm{~mm}$.
\end{abstract}

\section{Introduction}

Atrial fibrillation (AF) is one of the most common heart rhythm disorders and a leading cause of stroke. Radio-Frequency catheter ablation (RFCA) has become an accepted option for treating AF in today's electrophysiology (EP) labs, in particular, if drug treatment has become ineffective. RFCA of the pulmonary veins (PVs) is usually carried out under fluoroscopy guidance. Unfortunately, X-ray projection images cannot distinguish soft tissue well. To address this issue, fused visualization combining pre-operative high-resolution 3-D atrial CT and/or MR volumes with the fluoroscopic images has been developed (fluoro overlay image guidance) [1. Current fluoro overlay techniques are however usually static, i.e., they do not follow the heart while it beats and moves through the breathing cycle. This effect may impair the utility of fluoro overlays for catheter navigation. By synchronizing the fluoroscopic images with electrocardiogram (ECG), cardiac motion can be eliminated, and respiratory motion can be isolated for 
the fused visualization. While it has been widely recognized that motion compensation is crucial for fluoro overlays, image-based 3-D motion-compensation methods designed for EP applications are not yet known, mainly due to the fact that there are few discernible features in typical EP fluoro images. Motion compensated navigation for coronary intervention using magnetic tracking was suggested in [2, but it requires special catheters equipped with an electromagnetic sensor at increased cost. Only vertical motion in the imaging plane was compensated in 3] and [4] for liver embolization and hepatic artery catheterization, respectively. But the left atrium undergoes a three dimensional motion during respiration, as shown in [5]. This paper describes a novel image-based method to detect and compensate respiratory motion in $3-\mathrm{D}$ using a biplane $\mathrm{C}$ arm fluoroscopy device. Such an X-ray system has two imaging planes commonly referred to as A-plane and B-plane, respectively. To perform motion estimation, we track a commonly used EP catheter, the ring-shaped spiral catheter. This catheter is of unique shape and represents a good feature for tracking and motion estimation, as it is typically fixed at the ostium of the PV that is to be ablated.

\section{Three-Dimensional Lasso Model Initialization}

Our algorithm is based on the assumption that the perspective projection of a lasso catheter, when fit to the pulmonary veins, can be approximated as a 2-D ellipse. In turn, the lasso catheter is reconstructed in 3-D from biplane X-ray projections that are taken simultaneously using a calibrated biplane C-arm Xray system. The lasso catheter itself can be approximated as an ellipse in 3-D, because a 3-D elliptical object remains elliptical when perspectively projected onto a 2-D imaging plane. Under some special view orientations, the ellipse can, however, collapse to a line. As a consequence, our algorithm is designed to reconstruct a 3-D lasso model either from 2-D ellipses extracted from biplane X-ray views, or from one ellipse and one line (Fig. 11).

\subsection{Two-Dimensional Ellipse Extraction}

The projection of the lasso catheter on the imaging plane is first extracted by manual clicking followed by fast marching in one frame of the fluoroscopy sequence, as explained in [6]. A 2-D ellipse is then fitted using the method described

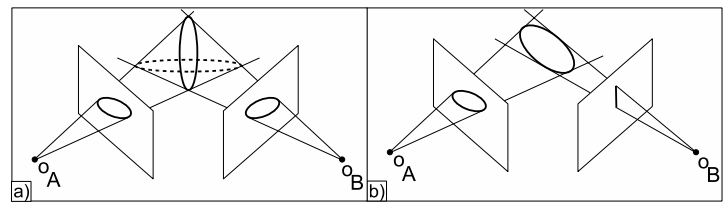

Fig. 1. 3-D lasso model initialization from two views. a) The 3-D model is found by the intersection of two 3-D elliptical cones. b) The model is found by intersecting the 3 -D elliptical cone with the plane determined by the $2-\mathrm{D}$ line and the optical center. 
in [7. Specifically, the method uses a least square estimation of the ellipse parameters $\mathbf{f}=(a, b, c, d, e, f)^{T}$ of the ellipse equation $a u^{2}+b u v+c v^{2}+d u+e v+f=0$ with the 2-D coordinates $u$ and $v$. Given a set of points $\mathbf{p}_{i}=\left(u_{i}, v_{i}\right)^{T}$ with $i=1, \ldots, N$, these can be combined in a measurement matrix $\mathbf{M}$ with the $i$-th row $\mathbf{M}(i,:)=\left(u_{i}^{2}, u_{i} v_{i}, v_{i}^{2}, u_{i}, v_{i}, 1\right)$ [ 8 . As the points may not necessarily lie exactly on the ellipse to be fitted, we are looking for the ellipse parameters $\hat{\mathbf{f}}$ that minimize $\hat{\mathbf{f}}=\arg \min _{\mathbf{f}}\|\mathbf{M f}\|_{2}^{2}$ subject to $\|\mathbf{f}\|_{2}=1$. Since the constraint $\|\mathbf{f}\|_{2}=1$ does not guarantee an elliptic solution, the method presented in 77 is used to assure the correct solution by enforcing the condition $b^{2}-4 a c<0$.

\subsection{Three-Dimensional Ellipse Reconstruction}

A 3-D point is projected onto an imaging plane as a 2-D point $\mathbf{p}=(u, v)^{T}$ by $\widetilde{\mathbf{p}}=$ $\mathbf{P} \cdot \mathbf{w}$. This equation holds in homogeneous coordinates where $\mathbf{w}=(x, y, z, 1)^{T}$ and $\widetilde{\mathbf{p}}=(u w, v w, w)^{T}$. The projection matrix $\mathbf{P} \in \mathbb{R}^{3 \times 4}$ is defined by $\mathbf{P}=$ $\mathbf{K}[\mathbf{R} \mid \mathbf{t}]$ with the matrix $\mathbf{K} \in \mathbb{R}^{3 \times 3}$ representing the intrinsic camera parameters, and the matrix $\mathbf{R} \in \mathbb{R}^{3 \times 3}$ and the vector $\mathbf{t} \in \mathbb{R}^{3}$ comprising the extrinsic camera parameters for rotation and translation, respectively. A 3-D elliptical cone can be spanned considering an imaging plane with the projection matrix $P$ and the ellipse within that plane parameterized by $\mathbf{C} \in \mathbb{R}^{3 \times 3}$. The base of the elliptical cone is the ellipse in the imaging plane and the vertex is the optical center that can be calculated as $\mathbf{o}=-\mathbf{R}^{-1} \mathbf{t}$. It can be shown that the elliptical cone can be represented by $\mathbf{Q}=\mathbf{P}^{T} \mathbf{C P}$ in matrix presentation 9 . For the regular case, the 3-D ellipse representing the 3-D lasso catheter is reconstructed by intersecting the two elliptical cones $\mathbf{Q}_{A}$ and $\mathbf{Q}_{B}$ corresponding to plane $A$ and plane $B$ of a biplane system respectively. The solution is found by calculating $\eta$ so that the quadric $\mathbf{Q}(\eta)=\mathbf{Q}_{A}+\eta \mathbf{Q}_{B}$ is of rank 2 ]. From a mathematical point of view, there are two feasible solutions, as illustrated in Fig. 1 a). In our application, we utilize our prior knowledge about the pseudo-circular shape of the lasso catheter and select the result that is more circular. For the special case where the lasso catheter is projected as close to being a line in one view, the method in 9 is not numerically stable. The special case is detected by considering the rank of the measurement matrix containing the first and second order point coordinates. A rank deficiency (ill-conditioning) indicates a linear dependency and therefore an ellipse that collapsed to a line. In this case, we propose to reconstruct the 3-D lasso model by the following procedures. First, a line is fitted to the line-like projection of the lasso catheter. It is calculated as the the principal axis of the points obtained by fast marching. Two arbitrary but distinct points $\mathbf{q}_{1}$ and $\mathbf{q}_{2}$ are then randomly selected on the fitted line and are connected to the optical center. The projection plane in which both the X-ray source and the fitted line lies is then determined by the two rays $\mathbf{q}_{1, o}$ and $\mathbf{q}_{2, o}$. In the second and final step, the 3-D lasso model is obtained by intersecting this plane with the elliptical cone defined by the ellipse from the second view. We propose the following method to calculate the analytical formulation of the conic section that intersects a plane $\widetilde{\mathbf{n}}=\left(x_{n}, y_{n}, z_{n}, d_{n}\right)^{T}$ with an elliptical cone $\mathbf{Q}$. The key idea is to transform the original coordinates, taken with respect to the iso-center of the $\mathrm{C}$-arm system, to 
new coordinates, in which the transformed $x$ - $y$-plane coincides with the cutting plane $\widetilde{\mathbf{n}}$. The transformation is given as

$$
\mathbf{S}=\left(\begin{array}{cccc}
\mathbf{u} & \mathbf{v} & \mathbf{n} & 0 \\
0 & 0 & d_{n} & 1
\end{array}\right)
$$

where $\mathbf{n}=\left(x_{n}, y_{n}, z_{n}\right)^{T}$ is normalized to unit length, and $\mathbf{u}$ and $\mathbf{v}$ can be any pair of unit length vectors lying in the cutting plane that are orthogonal to each other. $\mathbf{S}$ therefore presents a standard rigid-body transformation matrix after which the conic section $\mathbf{w}^{\prime}=\mathbf{S w}$ lies in the transformed $x$-y-plane with $z^{\prime}=0$. In addition, since

$$
\left(\mathbf{w}^{\prime}\right)^{T} \mathbf{U} \mathbf{w}^{\prime}=0 \quad \text { with } \quad \mathbf{U}=\left(\left(\mathbf{S}^{-1}\right)^{T} \mathbf{Q} \mathbf{S}^{-1}\right)
$$

and considering $z^{\prime}=0$, the parameters of the analytical formulation of the conic section can be obtained straightforwardly from the matrix $\mathbf{U}$ by $\hat{a}=u_{1,1}$, $\hat{b}=2 u_{1,2}, \hat{c}=u_{2,2}, \hat{d}=2 u_{1,4}, \hat{e}=2 u_{2,4}, \hat{f}=u_{4,4}$ where $u_{i, j}$ represents the element of the $i$-th row and $j$-th column of matrix $\mathbf{U}$. The model points in the transformed coordinate system are given as $\mathbf{w}_{i}^{\prime}$ (in homogeneous coordinates), $i=1, \ldots, L$, with the number of model points $L$, and are calculated using the estimated ellipse parameters $\hat{a}, \hat{b}, \hat{c}, \hat{d}, \hat{e}, \hat{f}$ as explained above. The 3 -D points for the model of the lasso catheter in the C-arm isocenter coordinate system, $\mathbf{w}_{i}$ (in homogeneous coordinates), are then calculated by $\mathbf{w}_{i}=\mathbf{S}^{-1} \mathbf{w}_{i}^{\prime}$.

\section{Three-Dimensional Lasso Model Tracking}

After the 3-D lasso model has been generated from the first frame of the fluoro sequence, it is tracked in 3-D throughout the remainder of the biplane sequence. To speed up the computational efficiency and to minimize the influence of peripheral structures that could interference with lasso tracking, the region of interest (ROI) for tracking is restricted to $400 \mathrm{X} 400$ pixels (on the $1024 \mathrm{X} 1024$ image) around the center of the tracked lasso catheter in the previous frame. Histogram equalization is further applied on the ROI to enhance image contrast. In addition, a vesselness filter [10] is used to enhance line-like structures such as the lasso catheter. The feature image is then binarized using Otsu's algorithm [1]. Finally, a distance map is calculated from the binarized image [12. A distance map encodes the distance from a point to its closed feature point, that is the nonzero point representing the extracted lasso catheter in our binarized feature image. The distance transform offers an important advantage. It provides a denoised representation of the fluoro image with a pronounced minimum around the 2-D shape of the lasso catheter. This way, we can still reach a good registration, even if we have a small 3-D model error, or if we start from a position that is somewhat away from the lasso catheter to be tracked. Lasso model tracking in $3-\mathrm{D}$ is achieved by performing $2-\mathrm{D} / 3-\mathrm{D}$ registration. To this end, the reconstructed 3-D lasso model is rotated by $\mathrm{R}$ and translated by $\mathrm{T}$ in $3-\mathrm{D}$ first. Then it is projected onto the two imaging planes of the biplane $\mathrm{C}$-arm system. The 

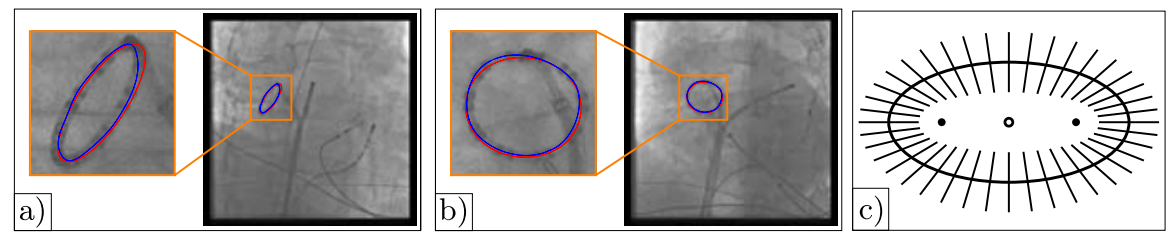

Fig. 2. a) Tracked ellipse (red) in plane A has an average distance to the manual segmentation (blue) of $1.0 \mathrm{~mm}$ (model error $0.5 \mathrm{~mm}$ ). b) In this particular frame, the tracking error $(0.6 \mathrm{~mm})$ is all due to the model error $(0.6 \mathrm{~mm})$. c) Ellipse with center (circle), focal points (dots) and normals.

average distance between the projected points and the closest feature point (i.e. the lasso catheter) in fluoroscopic images is efficiently calculated using the distance map introduced above. A suitable rotation and translation is found by optimizing

$$
\hat{\mathbf{R}}, \hat{\mathbf{T}}=\arg \min _{\mathbf{R}, \mathbf{T}} \sum_{i} \mathbf{I}_{\mathrm{DT}}\left(\mathbf{P}_{A} \cdot \mathbf{T} \cdot \mathbf{R} \cdot \mathbf{w}_{i}\right)+\sum_{i} \mathbf{I}_{\mathrm{DT}}\left(\mathbf{P}_{B} \cdot \mathbf{T} \cdot \mathbf{R} \cdot \mathbf{w}_{i}\right) .
$$

However, due to the fact that the shape of lasso catheters may not always be exactly elliptical, a simple elliptical 3-D model may not fit perfectly. To still obtain robust tracking, the distance of a forward projected 3-D point to the closest feature point is calculated as the smallest distance among all the points along the normal direction within five pixels from the projected point. An illustration of the normals to an ellipse is given in Fig. 22 c). A best neighbor optimizer is used to iteratively optimize the translational and rotational parameters. Registration is performed in two steps. In the first step, only the translation is considered, whereas in the second step a fully six-dimensional optimization is performed. Two-step registration is implemented to increase performance by lowering the number of iterations required for optimization.

\section{Results}

We evaluated our algorithm by calculating the tracking error for each of the imaging planes throughout seven different clinical fluoro sequences that were acquired during EP procedures on an AXIOM Artis dBA C-arm system (Siemens AG, Healthcare Sector, Forchheim, Germany). The system was calibrated using the method presented in [13. We focus on a typical setup involving one lasso catheter and one ablation catheter. The presence of other structures should not decrease the accuracy of our method, because we use a unique elliptical structure for registration. Model generation was experimentally evaluated by adding Gaussian noise with a standard deviation of $5 \mathrm{~mm}$ to 2-D forward-projected (3-D) ellipse points. In this case, we found the average distance between the resulting 3 -D model and the initial 3-D model to be $3.2 \mathrm{~mm}$. To calculate the tracking 
Table 1. Average tracking error for the clinical sequences used. The last row shows an average over all the 7 sequences for plane $\mathrm{A}$ and plane B, respectively.

\begin{tabular}{|c|c|c|c|c|c|c|}
\hline \multirow{2}{*}{ No. } & \multicolumn{3}{|c|}{ Plane A } & \multicolumn{3}{c|}{ Plane B } \\
\cline { 2 - 7 } & Mean & Std. & Model Error & Mean & Std. & Model Error \\
\hline 1 & $1.0 \mathrm{~mm}$ & $0.3 \mathrm{~mm}$ & $0.8 \mathrm{~mm}$ & $1.0 \mathrm{~mm}$ & $0.4 \mathrm{~mm}$ & $0.5 \mathrm{~mm}$ \\
\hline 2 & $1.1 \mathrm{~mm}$ & $0.3 \mathrm{~mm}$ & $0.5 \mathrm{~mm}$ & $0.7 \mathrm{~mm}$ & $0.2 \mathrm{~mm}$ & $0.6 \mathrm{~mm}$ \\
\hline 3 & $0.9 \mathrm{~mm}$ & $0.3 \mathrm{~mm}$ & $0.4 \mathrm{~mm}$ & $0.9 \mathrm{~mm}$ & $0.4 \mathrm{~mm}$ & $0.3 \mathrm{~mm}$ \\
\hline 4 & $1.0 \mathrm{~mm}$ & $0.2 \mathrm{~mm}$ & $0.6 \mathrm{~mm}$ & $1.8 \mathrm{~mm}$ & $0.4 \mathrm{~mm}$ & $1.1 \mathrm{~mm}$ \\
\hline 5 & $1.1 \mathrm{~mm}$ & $0.2 \mathrm{~mm}$ & $0.8 \mathrm{~mm}$ & $2.1 \mathrm{~mm}$ & $1.0 \mathrm{~mm}$ & $0.7 \mathrm{~mm}$ \\
\hline 6 & $1.0 \mathrm{~mm}$ & $0.6 \mathrm{~mm}$ & $0.5 \mathrm{~mm}$ & $0.8 \mathrm{~mm}$ & $0.2 \mathrm{~mm}$ & $0.6 \mathrm{~mm}$ \\
\hline 7 & $1.0 \mathrm{~mm}$ & $0.2 \mathrm{~mm}$ & $0.5 \mathrm{~mm}$ & $1.1 \mathrm{~mm}$ & $0.2 \mathrm{~mm}$ & $0.7 \mathrm{~mm}$ \\
\hline$\Sigma$ & $1.0 \mathrm{~mm}$ & $0.5 \mathrm{~mm}$ & $0.6 \mathrm{~mm}$ & $1.0 \mathrm{~mm}$ & $0.5 \mathrm{~mm}$ & $0.6 \mathrm{~mm}$ \\
\hline
\end{tabular}

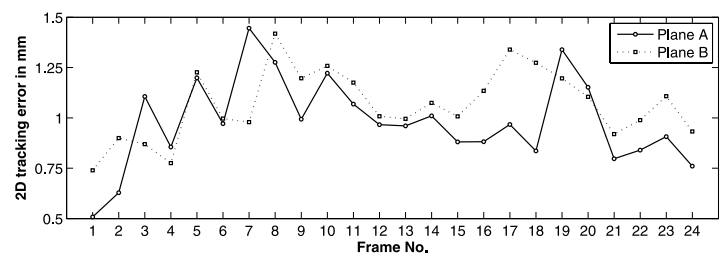

Fig. 3. Two-dimensional tracking error in mm for one sequence (No. 7) frame by frame. The A-plane and B-plane model errors are $0.5 \mathrm{~mm}$ and $0.7 \mathrm{~mm}$, respectively.

error, we forward projected the 3-D catheter model, computed from first frame, into both planes of the biplane imaging system after 2-D/3-D registration had been performed. In each frame, we calculated the average distance of the forward projected 3-D catheter model to a manually segmented lasso catheter. The manual catheter segmentation was supervised by a cardiologist, and we consider it our reference. This distance of the forward projected 3-D model to the reference was averaged over all frames of a particular sequence to arrive at an overall 2-D tracking error for each sequence. Both A-plane and B-plane tracking results for each of the seven test sequences are summarized in Table 1 . The mean average over the mean tracking errors was $1.0 \mathrm{~mm} \pm 0.5 \mathrm{~mm}$ for plane A and $1.0 \mathrm{~mm}$ $\pm 0.5 \mathrm{~mm}$ for plane B. An example for one frame of one sequence is given in Figs. 2 a) and b). Our method currently achieves a frame rate of 3 frames-persecond using a (single threaded) CPU implementation. Since this frame rate is clinically used for EP procedures to keep X-ray dose low, real-time tracking can be achieved in such a situation. In addition to the tracking error, a model error can be calculated. The model error can be obtained from the first image of a sequence as no registration was performed in this particular frame. A detailed frame-by-frame 2-D tracking error for sequence no. 7 is given in Fig. 3.

\section{Discussion and Conclusions}

Our experiments on clinical EP fluoro sequences show that the tracking error averages $1 \mathrm{~mm}$ including an average model error of $0.6 \mathrm{~mm}$. The real tracking 

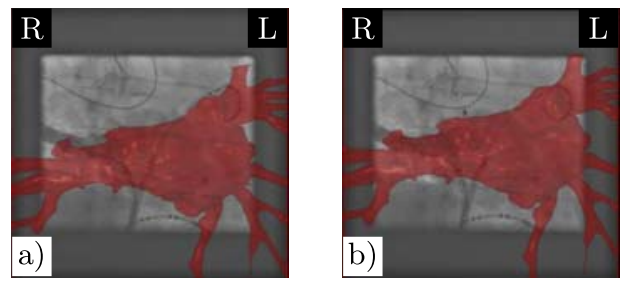

Fig. 4. a) Frame of a non-motion compensated sequence with fluoro overlay. b) The same sequence with motion compensation. Note how well both lasso catheter and contrast agent are matched to the morphology of the left atrium.

accuracy is therefore sub-millimeter. Considering that breathing motion in typical EP fluoro images is in the range of $15 \mathrm{~mm}$ and for some patients can be up to $40 \mathrm{~mm}$ for deep breathing, our method has the potential to significantly improve the accuracy of fluoro overlay images for EP navigation. To the best of our knowledge, this is the first image-based method that is specifically designed for the challenging task of 3-D breathing motion compensation during EP applications. The proposed method offers several advantages. First, it is workflow-friendly and does not require fiducial markers or additional contrast agent to be administered. Second, breathing motion is estimated directly in 3-D and right at the ablation site, meaning that motion estimation and compensation is essentially done in one step. There is no ambiguity coming from the inference of the real motion from surrogate motion estimates. Third, we estimate the motion online and update it constantly from fluoroscopy. Therefore we do not rely on a predefined motion model from which the real motion may deviate significantly during the procedure. Fourth, there are no restrictions for the 3-D data set that can be used as a fluoro overlay. In other words, the 3-D data set could come from MRI, CT or C-arm CT, e.g., syngo DynaCT Cardiac (Siemens AG, Healthcare Sector, Forchheim, Germany). Since the motion of the left atrium can be approximated by a rigid-body transform [5], it is possible to apply the motion estimate obtained by $3-\mathrm{D}$ catheter tracking to the static fluoro overlay. This way, we can obtain an animated version of our initial overlay that moves in sync with the real anatomy. Fig. 4 a) represents the conventional overlay technique without motion compensation, while Fig. 4 b) shows an animated fluoro overlay with motion compensation. With motion compensation, 3-D fluoro overlay and lasso catheter, fixed at the PV ostium, remain in sync. In addition, the contrast enhanced upper pulmonary vein, shown on the left side of the fluoro image in Fig. 4 b), remains well aligned with the corresponding vein of the volumetric data. Since simultaneous biplane imaging for EP applications is usually performed with both imaging planes set orthogonal to each other, tracking in 3-D should be even more accurate than suggested by our 2-D error analysis. 


\section{References}

1. Zagorchev, L., Manzke, R., Cury, R., Reddy, V.Y., Chan, R.C.: Rapid fusion of 2D x-ray fluoroscopy with 3D multislice CT for image-guided electrophysiology procedures. In: Medical Imaging 2007: Visualization and Image-Guided Procedures, San Diego, CA, USA, SPIE, vol. 6509, 65092B (2007)

2. Timinger, H., Krueger, S., Dietmayer, K., Borgert, J.: Motion Compensated Coronary Interventional Navigation by Means of Diaphram Tracking and Elastic Motion Models. Phys. Med. Biol. 50(3), 491-503 (2005)

3. Ross, J.C., Subramanian, N., Solomon, S.B.: Motion Correction for Augmented Fluoroscopy - Application to Liver Embolization. In: 5th IEEE International Symposium on Biomedical Imaging: From Nano to Macro. ISBI, pp. 1553-1556. IEEE Computer Society Press, Los Alamitos (2008)

4. Atasoy, S., Groher, M., Zikic, D., Glocker, B., Waggershauser, T., Pfister, M., Navab, N.: Real-time Respiratory Motion Tracking: Roadmap Correction for Hepatic Artery Catheterizations. In: Miga, M.I., Cleary, K.R. (eds.) Medical Imaging 2008: Visualization, Image-guided Procedures, and Modeling, San Diego, CA, USA, SPIE, vol. 6918, p. 691815 (2008)

5. Ector, J., Buck, S.D., Loeckx, D., Coudyzer, W., Maes, F., Dymarkowski, S., Bogaert, J., Heidbüchel, H.: Changes in Left Atrial Anatomy Due to Respiration: Impact on Three-Dimensional Image Integration During Atrial Fibrillation Ablation. Journal of Cardiovascular Electrophysiology 19(7), 828-834 (2008)

6. Liao, R., Xu, N., Sun, Y.: Location Constraint Based 2D-3D Registration of Fluoroscopic Images of CT Volumes for Image-Guided EP Procedures. In: Miga, M.I., Cleary, K.R. (eds.) Medical Imaging 2008: Visualization, Image-guided Procedures, and Modeling, SPIE, vol. 6918, p. 69182T (2008)

7. Halír, R., Flusser, J.: Numerically Stable Direct Least Squares Fitting Of Ellipses. In: Proceedings of the 6th Conference in Central Europe on Computer Graphics and Visualization, University of West Bohemia, Campus Bory, Plzen - Bory, Czech Republic, February 1998, pp. 253-257 (1998)

8. Hartley, R.I., Zisserman, A.: Multiple View Geometry in Computer Vision, 2nd edn. Cambridge University Press, Cambridge (2004)

9. Quan, L.: Conic Reconstruction and Correspondence From Two Views. IEEE Transactions On Pattern Analysis And Machine Intelligence 18(2), 151-160 (1996)

10. Sato, Y., Nakajima, S., Shiraga, N., Atsumi, H., Yoshida, S., Koller, T., Gerig, G., Kikinis, R.: 3D Multi-Scale Line Filter for Segmentation and Visualization of Curvilinear Structures in Medical Images. Medical Image Analysis 2(2), 143-168 (1998)

11. Otsu, N.: A Threshold Selection Method from Gray-Level Histograms. IEEE Transactions on Systems, Man, and Cybernetics 9(1), 62-66 (1979)

12. Breu, et al.: Linear time Euclidean distance transform algorithms. IEEE Transactions on Pattern Analysis and Machine Intelligence 17(5), 529-533 (1995)

13. Rougee, A., Picard, C.L., Trousset, Y.L., Ponchut, C.: Geometrical calibration for 3D x-ray imaging. In: Kim, Y. (ed.) Medical Imaging 1993: Image Capture, Formatting, and Display, Newport Beach, CA, USA, SPIE, February 1993, vol. 1897, pp. 161-169 (1993) 